\title{
Restless Legs Syndrome: clinical features, diagnosis and a practical approach to management
}

\author{
Subhashie Wijemanne, ${ }^{1}$ William Ondo ${ }^{2}$
}

${ }^{1}$ Department of Neurology, University of Texas Health San Antonio, San Antonio, Texas, USA

${ }^{2}$ Department of Neurology, Weill Cornell Medical School, Methodist Neurological Institute, Houston, Texas, USA

\section{Correspondence to} Dr Subhashie Wijemanne Departmentof Neurology, University of Texas Health San Antonio, San Antonio, Texas 78299, USA; wijemannesar@ uthscsa.edu

Accepted 29 September 2017

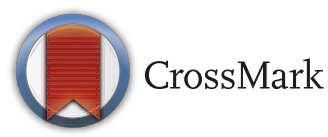

To cite: Wijemanne $S$, Ondo W. Pract Neurol 2017:17:444-452.

\begin{abstract}
Restless legs syndrome (RLS) is a chronic neurological disorder that interferes with rest and sleep. It has a wide spectrum of symptom severity, and treatment is started when symptoms become bothersome. Dopamine agonists and calcium channel apha-2-delta antagonists (gabapentin, gabapentin enacarbil and pregabalin) are first-line treatments; calcium channel alpha-2-deltas are preferred over dopamine agonists because they give less augmentation, a condition with symptom onset earlier in the day and intensification of RLS symptoms. Dopamine agonists can still be used as first-line therapy, but the dose should be kept as low as possible. Iron supplements are started when the serum ferritin concentration is $\leq 75 \mu \mathrm{g} / \mathrm{L}$, or if the transferrin saturation is less than $20 \%$. For severe or resistant RLS, a combined treatment approach can be effective. Augmentation can be very challenging to treat and lacks evidenced-based guidelines.
\end{abstract}

\section{INTRODUCTION}

Restless leg syndrome (RLS) is a chronic neurological disorder that interferes with rest and sleep, leading to poor quality of life and productivity. Sir Thomas Willis, a British anatomist and physician, first described the condition in 1685. In 1944, Karl-Axel-Ekbom, a Swedish physician who later became a pioneer in RLS research, described all the clinical features and coined the term RLS. ${ }^{1}$ In 1982, Akpinar first reported dramatic benefits of levodopa, ${ }^{2}$ and subsequently dopamine agonists became a mainstay of therapy. However, chronic dopaminergic treatment can aggravate symptoms in some people with RLS. This phenomenon, known as augmentation, has changed the way we approach therapy in RLS. ${ }^{34}$ Here we discuss a practical approach to the management of RLS.

\section{Clinical features}

RLS is a circadian disorder with unique clinical features (box 1). ${ }^{5}$ Symptoms typically develop in the evening or at night and can progressively worsen as the night progress but resolve by early hours of the morning. The symptoms occur after a period of relative inactivity. The key clinical feature of RLS is the irresistible urge to move the legs, either by itself or in response to uncomfortable paraesthesia of the legs. ${ }^{6}$ Moving the legs or walking improves the urge.

Patients often have difficulty articulating their symptoms and therefore may be reluctant to raise it with the physician. People use a wide range of descriptive terms to describe their leg paraesthesia, which include 'crawling', 'tingling', 'restless', 'cramping', 'creeping', 'pulling', 'painful', 'electric', 'tension', 'itching', 'burning' and 'prickly'. Isolated pain with an urge to move is not RLS. The urge to move the legs is sometimes experienced as a sense of intense anxiety or inner restlessness, and patients may not immediately recognise the accompanying urge to move the legs. Sometimes the sensory symptoms can be misleading: for example, the patient may misinterpret paraesthesia localised to the knee area as being 'arthritis' and may be reported to the doctor as such. Follow-up questions might reveal that the 'arthritis' appeared only in the evening, appeared after a period of rest, and improved with walking, satisfying the diagnostic criteria for RLS (box 1). Consider RLS in the differential diagnosis whenever a patient reports leg or sometimes arm discomfort that occurs in the evening or at night. A few focused questions will help to discern the correct diagnosis.

The leg paraesthesia commonly occur distal to the knee and back of the leg in the calf region (figure 1), but the 
Box 1 International Restless Legs Syndrome Study Group consensus diagnostic criteria for restless legs syndrome

1. An urge to move the legs usually, but not always, accompanied by, or felt to be caused by, uncomfortable and unpleasant sensations in the legs

2. The urge to move the legs and any accompanying unpleasant sensations begin or worsen during periods of rest or inactivity such as lying down or sitting

3. The urge to move the legs and any accompanying unpleasant sensations are partially or totally relieved by movement, such as walking or stretching, at least as long as the activity continues

4. The urge to move the legs and any accompanying unpleasant sensations during rest or inactivity only occur in the evening or night or are worse at night than during the day

5. The occurrence of the above features is not solely accounted for as symptoms primary to another medical or a behavioural condition (eg, myalgia, venous stasis, leg oedema, arthritis, leg cramps, positional discomfort and habitual foot tapping).

symptoms can affect areas such as the thigh and upper limbs. ${ }^{8}$ Most patients report that the paraesthesia appear to arise deep inside the leg such as the bones or the muscles and less commonly superficially near the skin. ${ }^{6}$ Symptoms typically involve both legs but commonly alternate between the legs and are rarely purely unilateral. The symptom severity can vary from a minor nuisance to an extreme debilitating state that completely disrupts sleep. The frequency of symptoms also varies from occasional to daily.

- Intermittent RLS is when symptoms, when not treated, have occurred on average less than twice weekly for the past year, with at least five lifetime events.

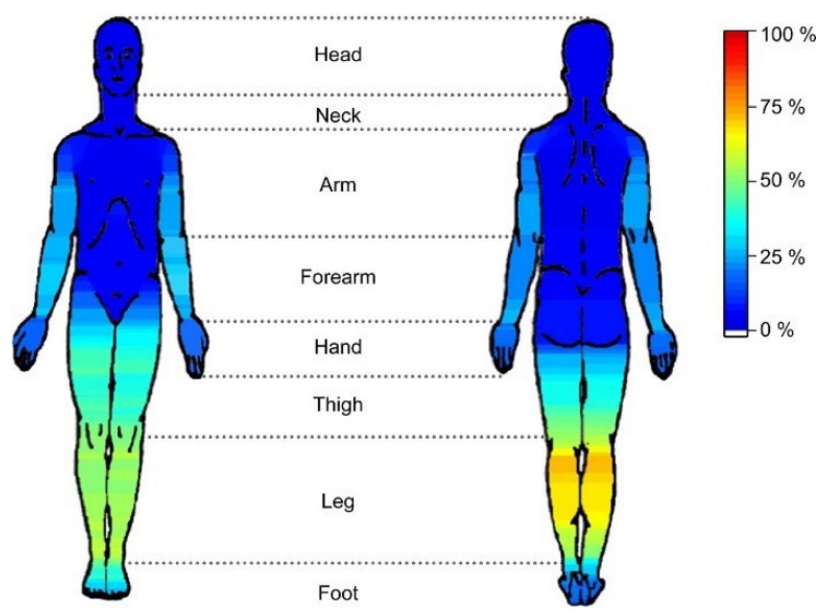

Figure 1 Body diagram showing sites of restless leg sensations. $^{8}$
- Chronic persistent RLS is when symptoms, when not treated, have occurred on average at least twice weekly for the past year.

RLS symptoms also show considerable day-to-day variability. There are few data on the long-term course of untreated patients with RLS; most studies report a gradual but irregular progression of symptoms.

Periodic limb movement of sleep (PLMS) is a highly stereotyped leg movement that develops in about $90 \%$ of patients with RLS. ${ }^{9}$ Its characteristic feature is extension of the big toe with partial flexion of the ankle, knee and sometimes the hip. Occasionally it involves the upper limbs. It typically involves both legs but not necessarily symmetrical or synchronously. If these movements occur while awake, it is known as 'periodic limb moments while awake'. PLMS is associated with increases in both heart rate and blood pressure, and the condition possibly increases the risk of developing cardiovascular disease. However, there is no conclusive association between RLS and cardiovascular risk. ${ }^{10} \mathrm{~A}$ polysomnogram can quantify PLMS, giving an indirect index of RLS severity. ${ }^{11}{ }^{12}$ However, having PLMS is not essential for the diagnosis of RLS, and thus an overnight polysomnogram is not needed for its clinical diagnosis.

The neurological examination is normal in primary RLS, although the clinician may see constant fidgety leg movements. It is rare to see 'periodic limb moments while awake' in the clinic, but one can often see them on patient home videos, along with PLMS. In secondary RLS, the neurological examination may show findings consistent with conditions associated with RLS, such as neuropathy, renal failure, pregnancy, Parkinson's disease and iron-deficiency anaemia.

\section{Diagnosis of RLS}

There are no objective tests or clinically available biomarkers for the diagnosis of RLS, which is based entirely on the subjective description of symptoms. The diagnostic criteria were revised in $2014 .^{5}$ These include five criteria that must all be present to diagnose RLS. The new fifth criterion states the need to exclude mimics of RLS, such as myalgia, venous stasis, leg oedema, arthritis, leg cramps, positional discomfort and habitual foot tapping. Although not included among the diagnostic criteria, clinical features that might further support a diagnosis of RLS include a family history of RLS, the presence of PLMS and a dramatic response to dopaminergic therapy. The diagnosis of RLS can be quite challenging in children, where it is often mistaken for growing pains or attention-deficit hyperactivity disorder.

The International Restless Legs Syndrome Rating Scale is frequently used in RLS studies. ${ }^{13}$ It comprises 10 questions, each with five responses scoring $0-4$ (total score $0-40$ ), with higher scores indicating more severe disease. This is a well-validated scale, and an 
improvement of more than three points is considered clinically significant. ${ }^{13} 14$

The RLS-6 scale has six items that assess daytime sleepiness, satisfaction with sleep and RLS severity when falling asleep, during the night, during the day when sitting or lying and during the day when active. ${ }^{15}$ Each item is rated on a scale of $0-10$. It is particularly useful because it assesses daytime and night-time symptoms separately.

\section{Epidemiology}

Most RLS studies have been in Caucasian populations and give a prevalence of 5\%-15\%. One large study reported an overall prevalence of $7 \%$ but clinically significant RLS in about $2.7 \% .{ }^{16}$ Women are affected twice as often as men, and the incidence increases with age. The prevalence is typically considered lower in Asian countries.

There are two common epidemiological groupings: early onset (younger than 45 years) and late onset (older than 45 years). Early-onset RLS has a peak incidence at 20-40 years of age, is often familial and has a slow disease evolution. Late-onset RLS may rapidly progress and is likely familial, and associated comorbidities are more common, especially iron deficiency.

With regards to RLS in children, a large paediatric population-based study involving over 10000 families in the USA and UK found a prevalence of definite RLS among children aged 8-11 years of $1.9 \%$ and among individuals aged $12-17$ years of $2.0 \% .{ }^{17}$

\section{Genetics}

RLS is a familial disorder, and particularly so the early onset primary RLS, which usually presents an autosomal dominant pattern of inheritance. About $63 \%$ of patients have at least one first-degree relative with the condition. Although there is a strong genetic contribution and numerous linkages identified, no highly penetrant gene has been identified. Published genomewide association studies (GWAS) have identified six different genes with single nucleotide polymorphisms BTBD9, MEIS1, PTPRD, MAP2K5, SKOR1 and TOX3 ${ }^{18}{ }^{19}$ Larger GWAS studies are are identifying additional involved genes. ${ }^{20}$ MEIS1 was the strongest risk factor for RLS. However, the variants in six genomic loci account for only a modest proportion of the genetically determined susceptibility to RLS. Thus, there are likely to be additional genetic contributors. MEIS1 has been implicated in limb development, raising the possibility that RLS has components of a developmental disorder. ${ }^{18}$

\section{Pathophysiology}

Iron, dopamine and opioid systems have been extensively studied to identify the physiological mechanisms underlying RLS. Although the serum iron is often normal, people with RLS have a state of low iron in the brain. Low brain iron has been demonstrated in neuropathological specimens, brain imaging and cerebrospinal fluid (CSF) evaluation. The brain areas most consistently showing this include the substantia nigra and, to a lesser extent, the putamen and caudate nucleus. Dysregulation of iron transport across bloodbrain barrier is one possible mechanism. ${ }^{21}$ The endothelial cells of the blood-brain barrier serve as an iron reservoir for the brain; the underlying problem in RLS probably is the lack of sufficient iron in reserve in the endothelial cells to meet physiological demands. ${ }^{21}$ The iron and dopamine systems in the central nervous system have multiple overlap, although their exact causal relationship in RLS is not known.

The dramatic response to dopaminergic medications implies a brain dopamine deficiency. However, animal and human studies of the dopamine system in RLS actually suggest a hyperdopaminergic state. The evidence for this is an increase in both 3-orthymethyl dopamine and the dopamine metabolite homovanillic acid in the CSF. It is postulated that in RLS, while total dopamine activity is likely heightened, there is circadian profile of dopamine activity that reflects hyperfunctioning in the morning and throughout the day, then relative hypofunctioning in the evening and nighttime. The dopamine receptor downregulation coupled with low dopamine activity at night may create a state of dopamine deficiency at night when RLS symptoms occur. ${ }^{22} 23$ The dopaminergic medications in the evening and night may correct for this relative evening decrease in dopamine, but this only exacerbates the situation in the long run by further downregulating the dopamine receptors. This could also explain the basis for augmentation. ${ }^{22} 23$ Specific alterations in dopamine- 1 receptors (compared with dopamine- 2 and dopamine- 3 receptors) with chronic dopaminergic therapy may also play a role. ${ }^{24}$

\section{Dopaminergic therapy and augmentation}

Half a century ago, there was no effective therapy for RLS. When the benefits of levodopa in RLS became known, this medication brought patients much welcome relief of symptoms. ${ }^{2}$ Subsequent multiple clinical trials confirmed levodopa's efficacy in RLS. ${ }^{25}$ However, as patients continued to use levodopa, the dose over time had to be increased to control worsening symptoms. It often reached a point where the symptoms were far worse than their baseline symptoms before starting levodopa treatment. This phenomenon, first described by Allen and Earley in $1996,{ }^{26}$ came to be known as augmentation.

Augmentation is characterised by a generalised increase in symptoms; this presents as a greater intensity of symptoms compared with before starting treatment, as well as symptoms appearing earlier in the day (box 2). ${ }^{27}$ For example, if symptoms used to appear after going to bed at night, with augmentation symptoms will start to appear earlier in the evening and with increased intensity. With further progression, 
Box 2 Max Planck Institute diagnostic criteria for augmentation

Following the use of a therapeutic agent, there is increase in symptom severity after initial therapeutic benefit, which is not accounted for by other factors, and is experienced on five out of 7 days during the previous week.

Earlier onset of symptoms for a minimum of 4 hours $0 R$, earlier onset of symptoms (between 2 hours and 4 hours) AND one of the following compared with symptom status prior to starting treatment.

- shorter latency to symptoms when at rest

- extension to other body parts

- greater intensity of symptoms

- shorter duration of relief from treatment.

the symptoms may appear in the afternoon or even in the morning, leading to a 24 -hour symptom cycle with loss of the circadian rhythm. Other features of augmentation include shorter latency of symptom onset after activity, to the point where activity will not suppress the RLS. The symptoms may also spread to other body regions.

Augmentation evolves slowly over time and may not be easily recognised. It may be mistaken for the natural progression of fluctuation of RLS. Stopping treatment for several weeks and observing for symptom improvement (or worsening) may help to distinguish between mild augmentation and natural progression. Clinicians should suspect augmentation whenever a patient who has been stable on dopaminergic medications requests an increase in dose. Augmentation must be distinguished from loss of efficacy or tolerance to treatment and also from medication rebound.

- Tolerance to treatment is loss of effectiveness of a medication over time but does not typically cause an earlier onset of symptoms.

- Medication rebound of symptoms is due to an end-ofdose effect, related to the pharmacokinetic properties of the medication, and commonly manifests as early morning worsening of symptoms.

Other causes of acute worsening of RLS, mimicking augmentation, include medications, sleep deprivation and iron deficiency.

The dopamine agonists ropinirole, ${ }^{2829}$ pramipexole ${ }^{30}$ 31 and rotigotine ${ }^{32} 33$ can give dramatic improvements in RLS symptoms and several large randomised placebo-controlled studies later confirmed their efficacy. These medications were approved for first-line therapy in RLS. The initial clinical trials were short and augmentation was not seen, so it was thought that augmentation was limited to levodopa. However, having used dopamine agonist for over 20 years for RLS, it is clear that augmentation is a major complication attributable to all dopamine agonists when used over a prolonged period of time. ${ }^{34-36}$ Most RLS referrals to a movement disorders specialist clinic are cases complicated by augmentation, which is a major therapeutic challenge. Augmentation is most common with levodopa, where it develops in $73 \%$ of patients. ${ }^{26}$ Pramipexole has an augmentation risk of $7 \%$ per year. ${ }^{36}$ The prevalence of augmentation accumulates fairly linearly with time, as reported in several retrospective studies. Over $50 \%$ of patients treated with pramipexole are estimated to experience augmentation in a little over 9 years. Rotigotine, a transdermal patch, may give less augmentation compared with an oral dopamine agonist, but we do not know if this simply results from continued exposure over 24 hours thus masking/treating the augmentation. ${ }^{37}$

\section{Management of RLS}

Currently, there is only symptomatic therapy for RLS; we await a curative or a disease-modifying agent. All patients can be offered non-pharmacological options. Certain medications aggravate RLS and a quick review of patient's drug history, including over-the-counter medications, will help to identify offending agents. These include antihistamines (especially sedating antihistamines that are often used as sleeping pills, such as diphenhydramine), dopamine-receptor blockers and serotonergic antidepressants, which mostly worsen PLMS.

Systemic iron deficiency can exacerbate RLS. Thus, all newly diagnosed patients should have their iron status checked, as should patients on stable therapy who report worsening symptoms. Optimising the iron status by itself may help to relieve RLS symptoms without any further intervention, although the response is often gradual. There is no diagnostic test to measure brain iron status (which is more relevant) available for routine clinical practice, and so we must rely on the serum iron concentration. This is done by measuring the serum ferritin concentration and ironbinding percentage. If the serum ferritin is $\leq 75 \mathrm{mg} / \mathrm{L}$ or the transferrin saturation is below $20 \%$, recommend iron therapy with oral iron supplements. Since ferritin is an acute phase reactant, it can be falsely elevated in certain underlying medical conditions. It also increases with age: as a rule, consider iron supplementation if the serum ferritin value (in $\mathrm{mg} / \mathrm{L}$ ) is less than the patient's age. Patients who cannot tolerate oral iron supplements may be considered for iron infusion; high-dose ferric carboxymaltose or low-molecular-weight iron dextran can be used (table 1). ${ }^{38}$ We have no strong evidence to support using iron sucrose in RLS, and it is probably not effective. ${ }^{39}$ Oral iron is also not well absorbed unless there is systemic iron deficiency; thus, counterintuitively, intravenous iron may be more indicated when the serum ferritin concentration is not very low.

Table 1 lists the commonly used medications in RLS, their minimal starting doses and the usual effective doses for RLS. Not all medications are approved by a regulatory agency. ${ }^{39}$ Patients with intermittent 


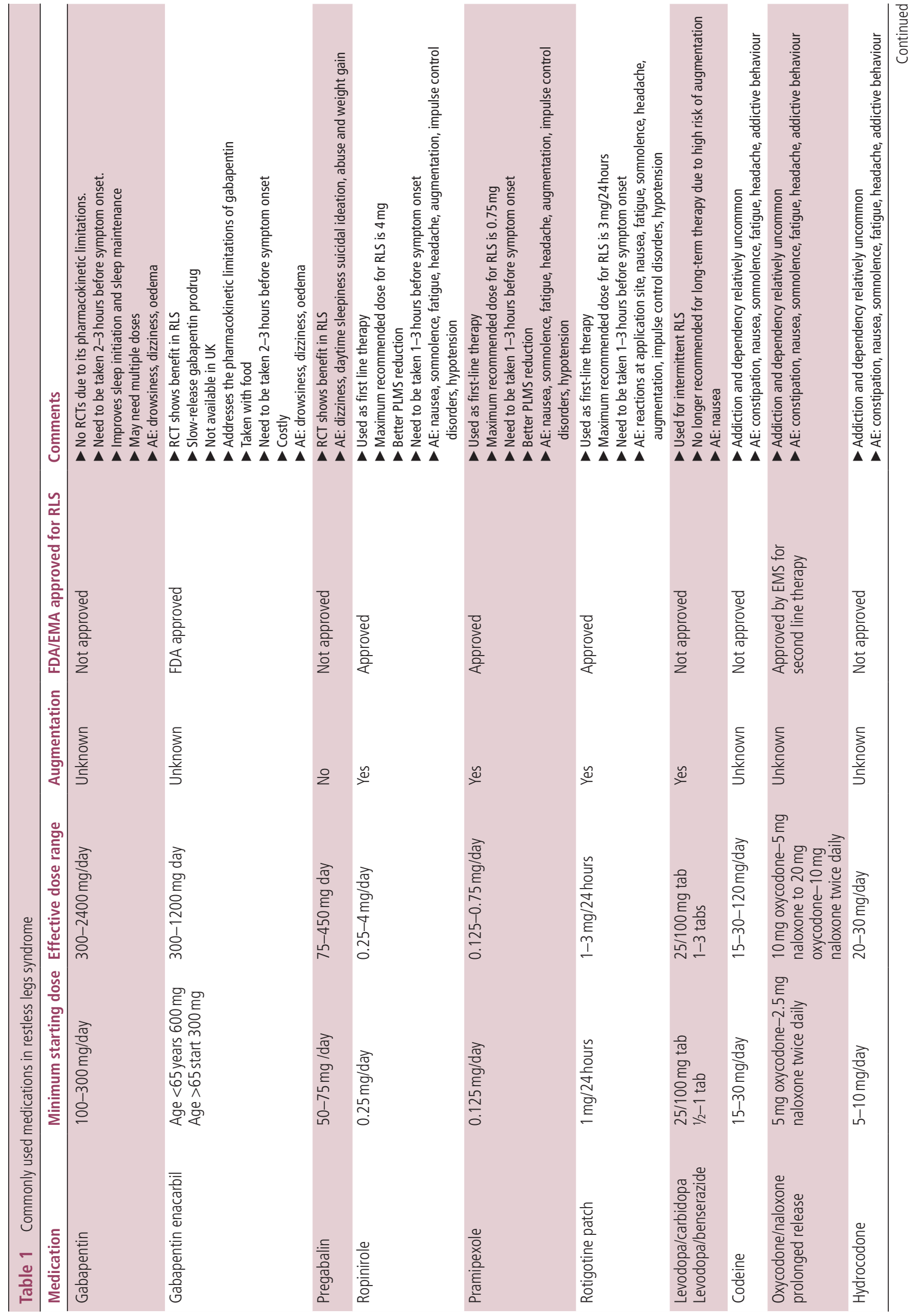

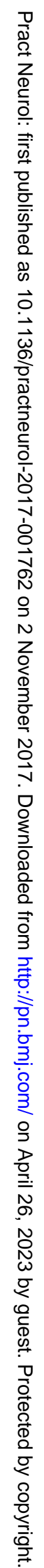




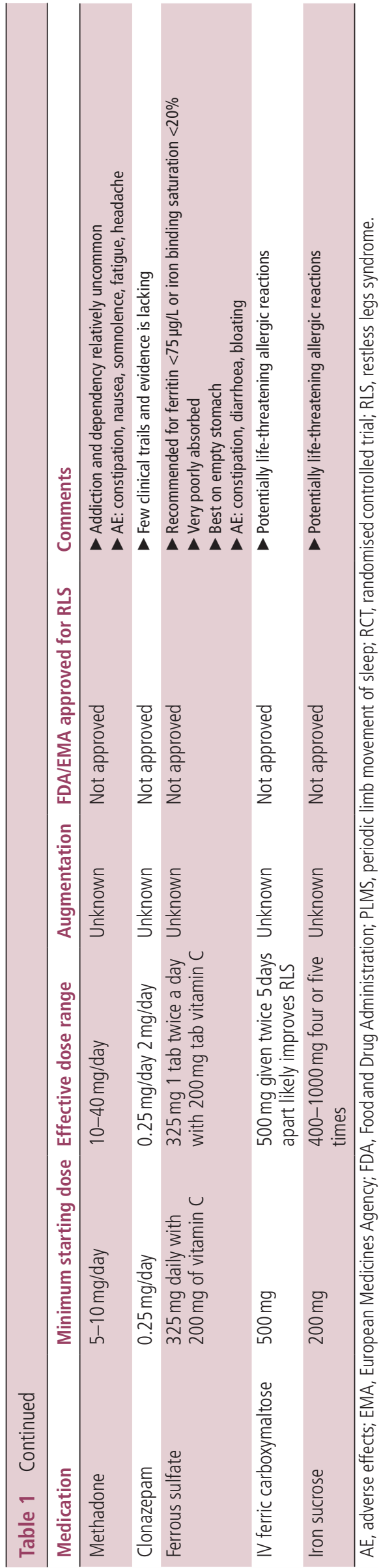

symptoms often have mild RLS and do not require medications. Oral dopamine agonists or calcium channel alpha-2-delta $(\alpha 2 \delta)$ ligands take 1-3 hours to become effective and therefore are not a practical solution for intermittent RLS. Opioids are typically reserved for moderate-to-severe RLS, but some patients are helped by a low potency opioid such as codeine, hydrocodone or tramadol. Daily treatment for RLS should only be started when symptoms are significantly impacting on quality of life in terms of frequency and severity; intermittent treatment might be considered in intermediate cases. ${ }^{4}$

In the past, dopaminergic agents were the recommended first-line treatment for chronic persistent RLS. However, augmentation concerns with dopaminergic agents has led researchers to look at new treatment options. A landmark clinical trial in 2014 compared $300 \mathrm{mg}$ of pregabalin (a calcium channel $\alpha 2 \delta$ ligand) against pramipexole for RLS. This study showed that pregabalin was more effective than pramipexole $0.25 \mathrm{mg}$ (but not $0.5 \mathrm{mg}$ ) and did not have the long-term complication of augmentation. ${ }^{40}$ However, the drop-out rate due to adverse events was higher with pregabalin. The other available calcium channel $\alpha 2 \delta$ ligands include gabapentin ${ }^{41}$ and gabapentin enacarbil ${ }^{42}$; this the only drug in this class officially approved for RLS. Gabapentin is shorter acting and has variable absorption but is inexpensive and often used. Patients may need multiple doses depending on the symptom onset and duration. Gabapentin enacarbil is an extended-release prodrug of gabapentin and so addresses the pharmacokinetic limitations of gabapentin. Its effectiveness has been shown in several double-blind clinical trials. The US Food and Drug Administration has approved gabapentin enacarbil at a dose of $600 \mathrm{mg}$ to be taken about 18:00 for the treatment of moderate-to-severe primary RLS in adults; however, it is not available in the UK. Its most common adverse events are sedation, dizziness, weight gain and oedema.

Given the high risk of augmentation with dopaminergic medications, a calcium channel $\alpha 2 \delta$ ligand is often preferred over dopamine agonists for longterm therapy. ${ }^{4}$ New guidelines were published in 2016 for the first-line treatment of RLS and prevention of dopaminergic augmentation. These guidelines encourage the use of calcium channel $\alpha 2 \delta$ ligands as first-line treatment in RLS over dopamine agonist. ${ }^{4} \mathrm{~A}$ dopamine agonist can be still used as first-line therapy but requires close follow-up to identify early signs of augmentation. The risk of augmentation is associated with higher doses and therefore it is important to keep the dose as low as possible. The recommended doses for RLS are much lower compared with doses used in Parkinson's disease (table 1); this is a common mistake by physicians who are not familiar with RLS therapy. Impulse control disorders such as pathological gambling, hypersexuality and compulsive 
shopping occur in 6\%-17\% of patients with RLS who take dopamine agonist, which is another concern with these medications. ${ }^{43}$

For severe RLS, opioids can be used as monotherapy or combination therapy. The benefits of oxycodone/naloxone prolonged release was shown in a randomised clinical trial, and so it is approved in many European countries as second-line therapy. ${ }^{44}$ Methadone is an option in those patients who fail dopaminergic medications or calcium channel $\alpha 2 \delta$ ligands. ${ }^{36}$ 45 Studies have shown that methadone, in contrast, shows neither augmentation nor major problems with continued efficacy after the first year of treatment. ${ }^{36}$ However, the current antiopioid climate can make these medicines difficult to use.

Benzodiazepines are sometimes used, but there is no strong evidence supporting their efficacy in RLS. ${ }^{46}$ They may help with insomnia associated with RLS.

A frequent mistake made in therapeutics is the incorrect timing of the chosen medication. Because of the pharmacokinetic properties, medications typically take time to become effective; for example, an immediate-release dopamine agonist may take 1-3 hours to become therapeutic. Therefore, for example, a patient experiencing symptom onset at 21:00 should take an initial dose by 19:00. Failure to give the medication at the appropriate time may be misinterpreted as medication failure. Early-onset RLS may require multiple small doses. Once the number of doses is determined, titrate to lowest dose that control symptoms. If monotherapy fails, combination therapy can be anecdotally effective. A low-dose dopamine agonist with either a calcium channel $\alpha 2 \delta$ ligand or an opioid can be tried.

\section{Management of augmentation}

There are essentially no prospective data to guide therapy for augmentation, and treatment recommendations are largely based on expert opinion. From a preventative standpoint, a calcium channel $\alpha 2 \delta$ ligand is a preferred over dopamine agonist as first-line therapy in a drug-naïve patient. If the patient is already taking a dopamine agonist and the symptoms are well controlled without augmentation, a decision can be made to either continue treatment with the dopamine agonist, provided that the dose is kept low without exceeding the recommended dose limit, or to switch to a calcium channel $\alpha 2 \delta$ ligand early in the course of management. This decision can be made on an individual basis.

If the augmentation is mild, the dose can be moved to an earlier time of the day to capture early symptoms or the dose can be split to be taken earlier. Another option is to transition to a longer acting drug such as rotigotine transdermal patch. ${ }^{47}$

For established problematic augmentation, the dopaminergic drug needs to be stopped, which is usually very difficult. There are different strategies:

\section{Key points}

- Consider restless legs syndrome (RLS) in the differential diagnosis whenever a patient reports leg or arm discomfort in the evening/night or insomnia.

- Augmentation due to dopaminergic medications is a major challenge in the long term treatment of RLS.

- Dopamine agonists and calcium channel $\alpha 2 \delta$ ligands are first-line therapy in RLS, but gabapentin derivatives are preferred over dopamine agonist since they do not cause augmentation.

- Dopamine agonists can still be used as first-line therapy, but the dose has to be kept low within the range recommended for $\mathrm{RLS}$, which is much lower than the doses used in Parkinson's disease.

- Check iron status in all newly diagnosed patients with RLS and in patients stable on medications who have worsening symptoms, and supplement with iron in those whose serum ferritin is $\leq 75 \mu \mathrm{g} / \mathrm{L}$ or whose transferrin saturation is $<20 \%$.

- One choice is to cross-titrate to a calcium channel $\alpha 2 \delta$ ligand and increase the dose of an opioid gradually to match the symptoms. The opioid can sometimes be withdrawn several weeks later. The goal is to stop the dopamine agonist completely and to maintain on the calcium channel $\alpha 2 \delta$ ligand.

- Another option is to stop the dopamine agonist completely without a cross titration. This will result in severe worsening of symptoms but for a shorter period, often 5-10 days. One reason to adopt this measure is that some patients after the washout period may no longer require therapy. If symptoms persist then a calcium channel $\alpha 2 \delta$ ligand can be started at that point. This method can be tried in a highly motivated patient since the symptoms can be unbearable during the transition period and result in severe sleep deprivation.

If one is cannot achieve good symptom control with the above methods, then the patient can continue with a low-dose opioid such as oxycodone or methadone. If the serum ferritin concentration is low and has not responded to or intolerant to oral iron, intravenous iron infusions can be considered. If the plan is to stop the dopaminergic drug, it is best to give intravenous iron 2-3 weeks beforehand, as it takes that long to have peak effect. There are no data available on how long complete resolution of augmentation takes, but in our experience, several weeks might be needed before symptoms return to baseline.

Contributors SW: drafting the work, revising it critically for important intellectual content, final approval of the version to be published and agreement to be accountable for all aspects of the work in ensuring that questions related to the accuracy or integrity of any part of the work are appropriately investigated and resolved. WO: revising it critically for important intellectual content.

Competing interests William Ondo: Speaker: UCBPharma Grant Support: Luitpold Pharmaceuticals. 
Provenance and peer review Commissioned; externally peer reviewed. This paper was reviewed by Jeremy Stern, London, UK.

(C) Article author(s) (or their employer(s) unless otherwise stated in the text of the article) 2017. All rights reserved. No commercial use is permitted unless otherwise expressly granted.

\section{REFERENCES}

1 Coccagna G, Vetrugno R, Lombardi C, et al. Restless legs syndrome: an historical note. Sleep Med 2004;5:279-83.

2 Akpinar S. Treatment of restless legs syndrome with levodopa plus benserazide. Arch Neurol 1982;39:739.

3 Wijemanne S, Jankovic J. Restless legs syndrome: clinical presentation diagnosis and treatment. Sleep Med 2015;16:678-90.

4 Garcia-Borreguero D, Silber MH, Winkelman JW, et al. Guidelines for the first-line treatment of restless legs syndrome/Willis-Ekbom disease, prevention and treatment of dopaminergic augmentation: a combined task force of the IRLSSG, EURLSSG, and the RLS-foundation. Sleep Med 2016;21:1-11.

5 Allen RP, Picchietti DL, Garcia-Borreguero D, et al. Restless legs syndrome/Willis-Ekbom disease diagnostic criteria: updated International Restless Legs Syndrome Study Group (IRLSSG) consensus criteria-history, rationale, description, and significance. Sleep Med 2014;15:860-73.

6 Yeh P, Ondo WG, Picchietti DL, et al. Depth and distribution of symptoms in restless legs syndrome/ Willis-Ekbom disease. $J$ Clin Sleep Med 2016;12:1669-80.

7 Ondo W, Jankovic J. Restless legs syndrome: clinicoetiologic correlates. Neurology 1996;47:1435-41.

8 Karroum EG, Leu-Semenescu S, Arnulf I. Topography of the sensations in primary restless legs syndrome. J Neurol Sci 2012;320(1-2):26-31.

9 Montplaisir J, Boucher S, Poirier G, et al. Clinical, polysomnographic, and genetic characteristics of restless legs syndrome: a study of 133 patients diagnosed with new standard criteria. Mov Disord 1997;12:61-5.

10 Kendzerska T, Kamra M, Murray BJ, et al. Incident cardiovascular events and death in individuals with restless legs syndrome or periodic limb movements in sleep: a systematic review. Sleep 2017;40.

11 Zucconi M, Ferri R, Allen R, et al. The official World Association of Sleep Medicine (WASM) standards for recording and scoring periodic leg movements in sleep (PLMS) and wakefulness (PLMW) developed in collaboration with a task force from the International Restless Legs Syndrome Study Group (IRLSSG). Sleep Med 2006;7:175-83.

12 Ferri R, Fulda S, Manconi M, et al. Night-to-night variability of periodic leg movements during sleep in restless legs syndrome and periodic limb movement disorder: comparison between the periodicity index and the PLMS index. Sleep Med 2013;14:293-6.

13 Walters AS, LeBrocq C, Dhar A, et al. Validation of the international restless legs syndrome study group rating scale for restless legs syndrome. Sleep Med 2003;4:121-32.

14 Allen RP. Minimal clinically significant change for the international restless legs syndrome study group rating scale in clinical trials is a score of 3. Sleep Med 2013;14:1229.

15 Kohnen R, Martinez-Martin P, Benes H, et al. Rating of daytime and nighttime symptoms in RLS: validation of the RLS-6 scale of restless legs syndrome/Willis-Ekbom disease. Sleep Med 2016;20:116-22.
16 Allen RP, Walters AS, Montplaisir J, et al. Restless legs syndrome prevalence and impact: REST general population study. Arch Intern Med 2005;165:1286-92.

17 Picchietti DL, Stevens HE. Early manifestations of restless legs syndrome in childhood and adolescence. Sleep Med 2008;9:770-81.

18 Winkelmann J, Schormair B, Lichtner P, et al. Genomewide association study of restless legs syndrome identifies common variants in three genomic regions. Nat Genet 2007;39:1000-6.

19 Winkelmann J, Czamara D, Schormair B, et al. Genomewide association study identifies novel restless legs syndrome susceptibility loci on 2p14 and 16q12.1. PLoS Genet 2011;7:e1002171.

20 Schormair B, Zhao C, Bell S, et al. 23andMe Research TeamDESIR study group. Identification of novel risk loci for restless legs syndrome in genome-wide association studies in individuals of European ancestry: a meta-analysis. Lancet Neurol 2017;16:898-907.

21 Connor JR, Ponnuru P, Wang XS, et al. Profile of altered brain iron acquisition in restless legs syndrome. Brain 2011;134(Pt 4):959-68.

22 Allen RP. Restless leg syndrome/Willis-Ekbom disease pathophysiology. Sleep Med Clin 2015;10:207-14.

23 Khan FH, Ahlberg CD, Chow CA, et al. Iron, dopamine, genetics, and hormones in the pathophysiology of restless legs syndrome. J Neurol 2017;264:1634-41.

24 Luo F, Li C, Ondo WG, et al. The long-term effects of the dopamine agonist pramipexole in a proposed restless legs syndrome animal model. Sleep Med 2011;12:41-6.

25 Trenkwalder C, Stiasny K, Pollmächer T, et al. L-dopa therapy of uremic and idiopathic restless legs syndrome: a doubleblind, crossover trial. Sleep 1995;18:681-8.

26 Allen RP, Earley CJ. Augmentation of the restless legs syndrome with carbidopa/levodopa. Sleep 1996;19:205-13.

27 García-Borreguero D, Allen RP, Kohnen R, et al. Diagnostic standards for dopaminergic augmentation of restless legs syndrome: report from a World Association of Sleep Medicine-International Restless Legs Syndrome Study Group consensus conference at the Max Planck Institute. Sleep Med 2007;8:520-30.

28 Trenkwalder C, Garcia-Borreguero D, Montagna P, et al. Ropinirole in the treatment of restless legs syndrome: results from the TREAT RLS 1 study, a 12 week, randomised, placebo controlled study in 10 European countries. J Neurol Neurosurg Psychiatry 2004;75:92-7.

29 Walters AS, Ondo WG, Dreykluft T, et al. Ropinirole is effective in the treatment of restless legs syndrome. TREAT RLS 2: a 12-week, double-blind, randomized, parallel-group, placebo-controlled study. Mov Disord 2004;19:1414-23.

30 Partinen M, Hirvonen K, Jama L, et al. Efficacy and safety of pramipexole in idiopathic restless legs syndrome: a polysomnographic dose-finding study--the PRELUDE study. Sleep Med 2006;7:407-17.

31 Oertel WH, Stiasny-Kolster K, Bergtholdt B, et al. Efficacy of pramipexole in restless legs syndrome: a six-week, multicenter, randomized, double-blind study (effect-RLS study). Mov Disord 2007;22:213-9.

32 Stiasny-Kolster K, Kohnen R, Schollmayer E, et al. Patch application of the dopamine agonist rotigotine to patients with moderate to advanced stages of restless legs syndrome: a double-blind, placebo-controlled pilot study. Mov Disord 2004;19:1432-8. 


\section{REVIEW}

33 Oertel W, Trenkwalder C, Beneš H, et al. Long-term safety and efficacy of rotigotine transdermal patch for moderate-tosevere idiopathic restless legs syndrome: a 5-year open-label extension study. Lancet Neurol 2011;10:710-20.

34 García-Borreguero D, Högl B, Ferini-Strambi L, et al. Systematic evaluation of augmentation during treatment with ropinirole in restless legs syndrome (Willis-Ekbom disease): results from a prospective, multicenter study over 66 weeks. Mov Disord 2012;27:277-83.

35 Högl B, Garcia-Borreguero D, Trenkwalder C, et al. Efficacy and augmentation during 6 months of double-blind pramipexole for restless legs syndrome. Sleep Med 2011;12:351-60.

36 Silver N, Allen RP, Senerth J, et al. A 10-year, longitudinal assessment of dopamine agonists and methadone in the treatment of restless legs syndrome. Sleep Med 2011;12:440-4.

37 Beneš H, García-Borreguero D, Ferini-Strambi L, et al. Augmentation in the treatment of restless legs syndrome with transdermal rotigotine. Sleep Med 2012;13:589-97.

38 Trenkwalder C, Winkelmann J, Oertel W, et al. Ferric carboxymaltose in patients with restless legs syndrome and nonanemic iron deficiency: A randomized trial. Mov Disord 2017.

39 Winkelman JW, Armstrong MJ, Allen RP, et al. Practice guideline summary: treatment of restless legs syndrome in adults: report of the guideline development, dissemination, and implementation subcommittee of the American academy of neurology. Neurology 2016;87:2585-93.

40 Allen RP, Chen C, Garcia-Borreguero D, et al. Comparison of pregabalin with pramipexole for restless legs syndrome. N Engl J Med 2014;370:621-31.

41 Happe S, Klösch G, Saletu B, et al. Treatment of idiopathic restless legs syndrome (RLS) with gabapentin. Neurology 2001;57:1717-9.

42 Lee DO, Buchfuhrer MJ, Garcia-Borreguero D, et al. Efficacy of gabapentin enacarbil in adult patients with severe primary restless legs syndrome. Sleep Med 2016;19:50-6.

43 Cornelius JR, Tippmann-Peikert M, Slocumb NL, et al. Impulse control disorders with the use of dopaminergic agents in restless legs syndrome: a case-control study. Sleep 2010;33:81-7.

44 Trenkwalder C, Beneš H, Grote L, et al. Prolonged release oxycodone-naloxone for treatment of severe restless legs syndrome after failure of previous treatment: a doubleblind, randomised, placebo-controlled trial with an open-label extension. Lancet Neurol 2013;12:1141-50.

45 Ondo WG. Methadone for refractory restless legs syndrome. Mov Disord 2005;20:345-8.

46 Carlos K, Prado GF, Teixeira CD, et al. Benzodiazepines for restless legs syndrome. Cochrane Database Syst Rev 2017;3:CD006939.

47 Trenkwalder C, Canelo M, Lang M, et al. Management of augmentation of restless legs syndrome with rotigotine: a 1-year observational study. Sleep Med 2017;30:257-65. 